\title{
Infancia, familia y políticas públicas: Tensiones y desafíos
}

\author{
Childhood, family and public policies: tensions and challenges
}

\author{
Nelson Zicavo Martínez \\ Universidad del Bío Bío
}

Recibido: $10-05-17$

Aceptado: $01-06-17$

\section{Resumen}

Se hace un análisis conceptual de las relaciones entre infancia, familia y políticas públicas, considerando las tensiones segregadoras que operan como obstáculos para avances cualitativos y cuantitativos de trascendencia; en particular respecto a la situación de niños, niñas y adolescentes. Se concluye que se requieren compromisos con el cambio y la ejecución de éste con base a políticas eficientes que ejecuten y respeten lo declarado en los convenios ratificados como derechos humanos.

Palabras clave: Infancia, familia, políticas públicas, derechos humanos, universalismo.

\begin{abstract}
A conceptual analysis of the relationships between childhood, family and public policies is made, considering the segregating tensions that operate as obstacles for qualitative and quantitative advances of transcendence; In particular with regard to the situation of children and adolescents. It is concluded that commitments are required to change and implement it based on efficient policies that implement and respect what is stated in conventions ratified as human rights.
\end{abstract}

Keywords: Childhood, family, public policies, human rights, universalism.

\footnotetext{
1. Universidad del Bío Bío, Chile. Email: nzicavo@ubiobio.cl
} 
Conocer a las familias y su conformación actual suele ser no solo un anhelo de las ciencias sociales, sino además un desafío para las políticas públicas de los diferentes gobiernos del mundo. Este grupo humano reside en el centro de la estructura social y constituye en sí mismo el espacio de producción y reproducción natural de las personas que la componen; de allí que comprender su organización, áreas, límites y fronteras, así como las dinámicas que la impulsan y las tensiones que la limitan, suele ser una meta compleja y anhelada para los gobiernos y estados de América Latina (también para las ciencias sociales), pues en base a ello se diseñan, organizan e inspiran las políticas públicas. Analizar su evolución y las tensiones reveladas por este proceso donde se incluye la concepción de los niños, niñas y adolescentes como sujetos de derechos, es el objetivo último de este trabajo.

\section{DESARROLLO DE TENSIONES Y POLÍTICAS PÚBLICAS SEGREGADORAS}

Tanto en Chile como en el resto de América latina, hemos visto en las últimas décadas, importantes esfuerzos de diversos organismos internacionales, así como de los gobiernos locales y la comunidad en general, en la búsqueda de logros cuyas metas son los equilibrios y la disminución de asimetrías de las inequidades económicas, políticas, sociales, alimenticias, de accesos, de justicia y educación, de las personas y las familias que ellas componen. Por sobre todo, destacan y resaltan los esfuerzos en políticas públicas basadas en los derechos humanos de las personas y en especial de los niños, niñas y adolescentes ${ }^{2}$, antes invisibilizados como sujetos sociales con identidad y decisiones trascendentes, con los cuales la sociedad debe contar y entender como parte indispensable de su orden estructural permanente. Este ha sido un enfoque significativo y trascendente que ha marcado una época sin retroceso, ubicando a la persona del NNA como sujeto de derecho de los esfuerzos por el desarrollo social con equidad.

No obstante, todos los significativos esfuerzos de los gobiernos latinoamericanos parecieron centrarse en los NNA con situaciones de vulneraciones significativas de sus derechos en la infancia (sobre todo temprana) teniendo una mirada no solo necesariamente proteccionista sino además y en cierto sentido, estigmatizando su realidad como personas carenciadas y con esto permitiendo la estratificación de los NNA como adecuados e inadecuados, con tipologías de personas diferentes, incluyendo diversos estratos socioeconómicos. De manera tal que al diseñar las intervenciones en sujetos percibidos como desajustados e incontrolables los respaldos estuvieran basados más en el control y el aislamiento social y afectivo (¿prevención?) que en la reinserción familiar y social. Como señala Saavedra:

\footnotetext{
2. En adelante se referirá como NNA.
} 
Los organismos públicos que delinean e implementan las políticas públicas orientadas a la infancia, han contribuido precisamente a que la forma de intervenir en situaciones de vulneraciones de derecho de alta complejidad, perpetúen el control sobre niños, niñas y adolescentes, las que aunque no aparecen como prácticas intencionales y planificadamente estigmatizadoras, poco a poco se van legitimando (Saavedra, 2009, p. 2)

Aquello es base importante para la Ley 20.286 (en Chile) de responsabilidad adolescente, más asentada en la criminalización de la pobreza en la infancia y de ciertos sectores vulnerables por su condición socioeconómica, que en la reparación social de sujetos que han carecido del apoyo social y familiar necesario, contribuyendo de esta manera "a la continuidad de la marginación de niños y niñas con graves problemas de salud mental, dificultando su acceso a prestaciones de salud que deben absorber las políticas radicadas en ese sector" (Saavedra, 2009, p. 2).

El control pareció ser la meta a través de la internación de NNA en lugares supuestamente- especializados ${ }^{3}$ en su atención, de esta manera aislándolos de una sociedad enferma que los afectó pero que desconoce su autoría ylos expulsa de su seno, renegando de ellos y poniendo el acento en la fuerza y no en la reeducación de los NNA con graves vulneraciones sociales y familiares, develando de esta manera tensiones significativas sin soluciones apropiadas.

En Chile existen elementos jurídicos que vienen a respaldar este abordaje desde el estado. Es así que -no casualmente-con el advenimiento de la democracia, durante 1990 el país se adscribe a la Convención Internacional de los Derechos del Niño (CDN), que impone el deber de garantizar el ejercicio irrestricto de los derechos humanos de los NNA allí plasmados tanto en el plano jurídico, como en el institucional y en el socioeconómico.

Más adelante y luego de un largo camino, Muñoz (2013) recuerda que durante el año 2012 se presenta un proyecto de Ley de Protección Integral para NNA que prometía dar respuesta a una mayor institucionalidad de la infancia en base al respeto de los derechos de toda la niñez y adolescencia del país, compromiso que aún se encuentra sin el cumplimiento debido en cuanto a integralidad de su aplicación. Este proyecto supone la superación de una visión reduccionista de los NNA limitada a su vulnerabilidad y a la vez intenta el reconocimiento activo al carácter universal de la protección, garantizando los accesos indispensables a educación, salud, alimentación, inclusión social y cuidados para su desarrollo y crecimiento adecuado. Dicho proyecto pretendió poner el acento en que los derechos de los NNA sean exigibles frente a las personas y organismos con que se vinculan, sea familia, estamentos educacionales, administrativos o judiciales.

\footnotetext{
${ }^{3}$ En la actualidad los continuos escándalos de abusos y violencia al interior de estos establecimientos han llevado a desmantelarlos paulatinamente y replantearse el eje de atención y la política propiamente tal.
} 
Hay aquí una búsqueda activa en resguardar no solo la restitución de derechos de los NNA vulnerados, sino a la vez garantizar derechos universales a todas las personas comprendidas en esta categoría, nos referimos al derecho a la no discriminación, a las posibilidades de desarrollo adecuado y a la supervivencia en condiciones aceptables, es en definitiva poner de relieve el interés superior del niño como orientación cimera de las políticas públicas.

\section{DERECHOS HUMANOS Y UNIVERSALISMO}

Lo anterior supone no solo el reconocimiento de dichos derechos que obligan al estado, sus instituciones y personas, sino además garantiza el efectivo ejercicio de los mismos con indicadores claros que permitan la supervisión de su implementación, solo entonces se podría hablar de explícitamente de derechos universales.

Efectivamente, el proyecto significa un avance cualitativo de trascendencia, no obstante sigue siendo limitado según pues si bien, por un lado, reconoce que los NNA son sujetos de derecho y al mismo tiempo depositarios de una creciente autonomía que llevarán adelante en función de su desarrollo así como de la madurez, de todas maneras "da cuenta de un énfasis punitivo en el tratamiento de esta población, que aparece catalogada más bien como delincuente y no como sujeto de derechos en proceso de desarrollo" (Muñoz, 2013, p. 4)

De todas maneras, resulta significativo que en el instrumento referido, se consagren derechos universales claramente delineados, tales como:

el derecho a la vida y a la integralidad física y psíquica, a ser cuidados por la familia de origen y/o a tener una relación directa y regular con los padres; a ser oídos y que su opinión sea tomada en cuenta de acuerdo a su edad y madurez; a la libre expresión; a la libre asociación; a la convivencia familiar; a la salud y a la educación; a no ser discriminados; al descanso y esparcimiento; a su protección contra todas las formas de explotación o abusos (Muñoz, 2013, p. 7).

Tal meridiana claridad no solo es orientadora, sino que obliga a las políticas públicas a construir y generar acciones que establezcan acciones y conductas sociales trasparentes con garantías reales basadas en derechos humanos universales para todos los NNA.

Es en este sentido, es muy importante superar los esfuerzos sectoriales -a menudo parcializados en sus políticas- sustituyéndolos por la intencionalidad y concreción de políticas interinstitucionales que puedan dejar atrás los lineamientos verticalistas a espaldas de las regiones o de los actores ciudadanos y que estaban basados en políticas centralistas con imágenes de NNA desajustados y 
criminalizados, a veces incluso hasta segmentados territorialmente, categorizando y discriminando personas.

Resulta trascendente que Chile asuma que el camino generador de nuevos abordajes para la protección de NNA debe producirse como un abordaje universalista, ya que no es posible dar cuenta de los objetivos planteados solo con una reflexión a puertas cerradas dada por organismos especializados, pues "la definición que como país estamos llamados a realizar al momento de definir políticas integrales, fundamentadas en el enfoque de derechos humanos, es una tarea que debe incluir a todos los servicios públicos" (Saavedra, 2009, p. 10).

Este abordaje basado en la universalización, en la integración de los diversos esfuerzos institucionales (SENAME, Chile Crece Contigo, SERNAM, SENAMA, entre otros) supone y obliga acciones de consensos entre todos los actores institucionales para poder optimizar intervenciones y a la vez combatir la inadecuada idea que asocia vulneración y peligrosidad de los NNA. El centro de dichas políticas, debiera ser un sistema de protección que fuera abordado con carácter integral tanto en lo social como en lo económico con el fin de cuidar y respaldar el ejercicio de los derechos centrados en las personas.

Es por eso que la intersectorialidad, la universalización, es base indispensable para la integralidad de modelos de gestión en el abordaje articulado de diversos esfuerzos provenientes de diversas áreas del servicio público con el acento puesto en el desarrollo de las personas. No obstante, al parecer aun nos enfrentamos más a una aspiración que una realidad, estamos ante una tarea pendiente.

La vetusta política asistencialista no ha sido más que un conjunto de (posiblemente) medidas necesarias históricamente ${ }^{4}$ basadas en el traspaso de bienes y servicios a los desposeídos, a las personas fuertemente carenciadas, concebidos como sujetos receptores de políticas y no creadores activos de las mismas. Ello permitió concebir a la persona como objeto de políticas y no como sujeto de derechos, tal es el cambio epistémico que se comienza a observar paulatinamente en las últimas décadas.

Sin embargo, transitar desde el asistencialismo a la política social basada en los derechos humanos de la persona, supone "avanzar hacia regímenes de política social que aseguren niveles básicos de universalismo en materia de política social" (Andrenacci y Repetto, 2006, p. 2).

\footnotetext{
4. Resulta inevitable referirse someramente -más resultaría impropio quedarse en dichos límites- a la época histórica donde la lucha contra la pobreza fue el objetivo prioritario para la generación de condiciones de vida apropiadas y dignas, así como la reducción de las desigualdades en todo ámbito, situación heredada de regímenes dictatoriales centrados en el éxito del capital y donde las políticas sociales fueron relegadas al olvido o fueron acalladas con la fuerza del poder. La década del 70 criminalizó la pobreza y extremó la estratificación de los accesos.
} 
La actualización de las políticas públicas universalistas con base en los derechos humanos, intenta resignificar las prioridades de la política social retirando el acento desde la contención pacificadora de los desposeídos de las décadas del 70 y 80 , hasta la inclusión social, aunque esta seaaún parcial y muy estratificada, con duras exclusiones y fuertes desigualdades de la ciudadanía.

El horizonte del universalismo, como objetivo de politica social, parte del diagnóstico según el cual el problema social más importante de América Latina es la dimensión de la desigualdad. La construcción de igualdad social implica la construcción de sociedades económicamente integradas, socialmente cohesivas y politicamente compartidas, donde las diferencias no configuren segmentaciones (Andrenacci y Repetto, 2006, p. 15).

Se observa entonces que las políticas públicas universales están llamadas a ser significativas en la creación y consolidación de equidad ciudadana y territorial, debieran ser parte esencial en la contribución y fortalecimiento de los imaginarios sociales e individuales de equidad y solidaridad entre las personas, familias, instituciones y territorios, se trata pues, de construir sociedades y economías más justas con atención a la equidad. Para ello se requiere de un estado vigilante $\mathrm{y}$ atento al cumplimiento de sus directrices en derechos humanos vinculantes, que propenda a un estado de derecho donde el acento este puesto en la familia y la equidad de sus integrantes como sujetos de derecho, sobre todo, pero no únicamente, para los NNA.

Sobre esto, deja meridana claridad el cambio de paradigma de la ONU declarado en la Convención Internacional de Derechos del Niño (1989), donde seasume explícitamente que los NNA son legítimos sujetos de derecho. Esto implica que los Estados firmantes están obligados a garantizar la satisfacción de los derechos de NNA y por tanto tienen que diseñar políticas públicas que garanticen tales cumplimientos. Además, demanda la necesidad imperativa de que las mismas se lleven efecto de manera eficiente y establece mecanismos de supervisión e indicadores que den fe del cumplimiento de las mismas.

\section{LA FAMILIA Y LOS DERECHOS DE LOS NNA}

Es en este escenario, que cobran presencia significativa durante la última década los derechos humanos de los NNA inmersos en el seno de la familia, ya que el acento se sitúa en el derecho de los NNA a la alimentación adecuada, al cuidado oportuno, a ser protegidos y a vivir en familia (entre otros). Estas personas tienen derecho a un estándar adecuado de vida en cuanto a alimentación, vestimenta, vivienda y a la mejora paulatina y continua de sus condiciones de existencia. Ello exige varios garantes de tal desarrollo, por un lado el estado, que debe promover las acciones e intersectorialidad necesarias para el éxito de sus políticas y a la vez garantizar que tal derecho no sea vulnerado en su esencia; por otro la familia, 
que tiene responsabilidades que no pueden ni deben ser eludidas pues debe asumir claramente que los NNA tienen derecho a ser escuchados en sus intereses, opiniones, deseos y necesidades, tienen derecho a criarse en un espacio familiar que garantice el acceso a la alimentación, afecto y cuidados apropiados, promoviendo y garantizando su desarrollo armónico en paz e igualdad de oportunidades.

Hacer exigibles los derechos de los NNA constituye una tarea aún pendiente para la gran mayoria de los Estados ya que las medidas implementadas han sido por demás insuficientes. En general, el goce de los derechos de los niños se ve restringido por diversos obstáculos que son de naturaleza cultural, legal, económica y financiera. Es necesaria la adopción de medidas de todo tipo para poner en práctica los derechos reconocidos y en particular romper con la lógica tutelar que ha primado en la normativa, instituciones y prácticas estatales (Pautassi y Royo, 2012, p. 18).

Más adelante señalan:

Todas las politicas que atañen a la infancia deben incorporar un enfoque basado en los derechos de la infancia. Es conveniente enfatizar una y otra vez, que este planteamiento descansa en el reconocimiento del niño como titular de derechos y no como beneficiario de la benevolencia de los adultos (Pautassi y Royo, 2012, p. 20)

Ser reconocidos como personas titulares de derechos implica que la sociedad, el estado (sus instituciones) y las familias reconozcan y asuman activamente algunos principios ineludibles que están en la base de tal concepción, son principios trascendentales que hay que tener en cuenta para este trabajo, son los reconocidos por la CDN como los principios de no discriminación, el interés superior del niño, el derecho de toda persona a la vida, el derecho a la supervivencia y al desarrollo apropiado, el derecho de losNNA a expresar sus opiniones y a que ser tenidos en cuenta como sujetos independientes e individuales con derechos propios e inalienables. Son trascendentes pues en ellos se asienta el respeto al derecho de los NNA o,en caso opuesto, pueden desdibujarse bajo la sombra de la intencionalidad familiar de crianzas inadecuadas, con tal propósito consciente o aun sin percatarse, pero igualmente limitante.

Resulta indudable que los NNA requieren de recursos económicos que vayan a garantizar el acceso a una nutrición balanceada y adecuada para satisfacer las necesidades derivadas del crecimiento y desarrollo de su vida,más aún también tienen derecho a ser cuidados y a vivir en familia independientemente del lazo contractual de sus padres. Cada uno de estos ángulos de evaluación tienen trascendencia en el desarrollo de su vida, no solo de comida se alimenta el desarrollo de una persona ya que el bienestar también depende de la armonía y el desarrollo afectivo, lo cual se garantiza con el acceso a ambos padres de manera 
equitativa. La labor de crianza no solo es un derecho del niño también es deber y derecho de ambos padres (Ferrari y Zicavo, 2011).

Sin embargo, la labor de crianza ha sido asignada socioculturalmente al mundo femenino basada en la división sexual del trabajo y en la naturalización patriarcal de concebir a la familia como el ámbito obligatorio de procreación y cuidado de los niños a cargo de las mujeres, sean estas madres, hermanas o colaboradoras internas o externas al medio familiar, dejando fuera de las obligaciones de crianza al mundo masculino al cual se le asigna el rol de proveedor (Ferrari y Zicavo, 2011).

Resulta entonces notorio que al visibilizar el trabajo derivado de la crianza de los hijos, la sociedad enfrenta un escenario donde revelar las inequidades es a la vez imperioso y de cuidado, ya que resulta evidente que es un espacio de desigualdad donde las políticas públicas reman en una dirección y avanzan en sentido opuesto. Parece ser que, promover la participación masculina es inclusiva no solo con los derechos de los NNA sino además con los femeninos y al mismo tiempo de los hombres, que desean paternar cada vez más. Pero las políticas públicas actuales, marginan lo masculino de estos espacios que son re-feminizados constantemente a través de los limitados permisos parentales para cuidar, con notoria inequidad respecto de tiempos femeninos y masculinos dados por la legalidad laboral vigente (Ferrari y Zicavo, 2011). Se posee una intencionalidad de espíritu en la ley que se borra con la realidad cotidiana laboral.

Es indispensable tener en cuenta que, "el contar con la consideración del derecho al cuidado universal será un primer paso para distribuir las responsabilidades de cuidado en todos los miembros de la sociedad y no sólo en las mujeres. Con ello se comparten las responsabilidades alimentarias y nutricionales" (Pautassi yRoyo, 2012, p. 32).

Así, el Consenso de Quito asume como compromiso "formular y aplicar políticas de Estado que favorezcan la responsabilidad compartida equitativamente entre mujeres y hombres en el ámbito familiar, superando los estereotipos de género, reconociendo la importancia del cuidado y del trabajo doméstico para la reproducción económica y el bienestar de la sociedad como una de las formas de superar la división sexual del trabajo. Asimismo se acuerda adoptar medidas en todas las esferas de la vida particular, en los ámbitos económico y social, incluidas reformas institucionales, para garantizar el reconocimiento y el aporte al bienestar de las familias y al desarrollo, promover su inclusión en las cuentas nacionales (Pautassi y Royo, 2012, p. 33).

Como podemos observar, si bien los instrumentos se encuentran presentes, se hace imprescindible no solo un cambio cultural sino al parecer, la toma de 
decisiones tangibles por parte del estado para que tal aspiración se convierta en realidad y fuente de equidad, en derechos humanos. Por su parte "la CDN establece $^{5}$ que corresponde al Estado garantizar el reconocimiento del principio que ambos padres tienen obligaciones comunes en lo que respecta a la crianza y el desarrollo del niño (...) su preocupación principal será el interés superior del niño...” (Pautassi y Royo, 2012, p. 33).

No obstante, es preciso formularse una pregunta básica: ¿las leyes laborales promueven la paternidad responsable y la equidad de los accesos de los niños a madre y padre?

La respuesta que de allí emana orienta respecto de la promoción real de los derechos humanos de los NNA. Resulta notorio que existe un acceso diferencial respecto de las licencias parentales para mujeres y hombres, permisos para cuidar que se promueven en la políticas públicas y que de alguna manera visibilizan una realidad que se intenta negar: la licencia maternal para el cuidado de los nuevos nacimientos de la pareja es abrumadoramente mayor en mujeres (más de 10 veces) que en hombres emitiendo de esta manera un mensaje asimétrico con atención a inequidades concretas: por un lado se promueve y desea la corresponsabilidad parenta $l^{6}$ así como el derecho de los NNA a contar con padre y madre disponibles en todo momento (física y afectivamente) y, por otro lado, es imposible que esto se cumpla pues la ley no lo ampara. Se asume indispensable la presencia masculina en el crecimiento y desarrollo de los NNA sin embargo no se "toca" la presencia biologicista al cuidado de los hijos como norma imperante (aunque desfasadamente patriarcal). Eso pareciera marcar rumbos claros... aunque en esencia sean obscuros.

Desde sus inicios las licencias parentales fueron concebidas -en la década del 70- para promover la paternidad masculina responsable, porque se entiende que beneficia a todos los actores y por cierto por ser un derecho humano, para los niños, para los padres y para las madres (Ferrari y Zicavo, 2011).

Sin embargo, es claro que las políticas maternalistas promueven y elevan a su máxima categoría la importancia de los cuidados sobre los hijos, así como las capacidades femeninas para la crianza de los NNA. Resulta claramente observable que históricamente:

Un piso maternalista es esencial para proteger a las mujeres en su condición de madres y a sus propios hijos (de ambos progenitores). Sin embargo, las politicas maternalistas pueden afectar a la equidad de manera contradictoria. Si bien dichas politicas reconocen públicamente y apoyan

\footnotetext{
5. En el artículo 18 inc. 1

6. El cuidado de los hijos no solo es un derecho de los NNA sino también de ambos padres, y trae aparejado como efecto resultante, la posibilidad de desarrollo de femenino también en otros ámbitos de la vida pública.
} 
la maternidad como dimensión central en la vida de las mujeres, elevando entonces el estatus maternal, refuerzan a la vez la noción de que el cuidado es únicamente responsabilidad femenina. Es materia empírica establecer cuáles políticas maternalistas promueven también la equidad de género al emparejar la cancha en lugar de reforzar la división sexual del trabajo entre hombres y mujeres" (Blofield yMartínez, 2014, p. 6).

Sin dudas es algo a tener en cuenta por las políticas públicas para avanzar en la dirección correcta, pues las tensiones son más que evidentes. Si bien el maternalismo fue históricamente necesario, en la actualidad los derechos humanos de los NNA requieren de corresponsabilidad parental y estatal. Se deben redistribuirlas responsabilidades de los cuidados de los NNA en ambos padres, la familia y el Estado. La corresponsabilidad no solo es paternal sino también familiar y Estatal.

Entonces, se debe visibilizar que ambos padres (y el Estado por supuesto) poseen el derecho y deber de cuidar y promover al desarrollo de sus hijos, así como también es un derecho de los NNA mantener una relación directa y regular con la madre y con el padre, impedirlo implica alterar el proceso y vulnerar dichos accesos.

Es necesario subrayar que "si las formas que asume la resolución de la seguridad alimentaria de los NNA son a costa del incremento del trabajo de las mujeres, lejos estará de resolverse desde un enfoque de derechos humanos" (Pautassi y Royo, 2012, p. 37). Este asunto trascendente para las políticas públicas de las naciones latinoamericanas. Resulta imprescindible que se cambie el foco desde lo maternal a lo familiar, integrando responsabilidades compartidas, de esa manera se acabará con las ayudas que perpetúan las brechas existentes maquillando hipócritamente las asimetrías supuestamente novedosas.

Más aun, resulta imprescindible que se camine a un futuro donde se puedan conciliar o dar equilibrio a la vida familiar y la dedicación laboral, en ese espacio las políticas públicas del estado serán actores trascendentes. Resulta imperativo que los cuidados de la familia no sean únicamente un asunto femenino, no solo para los NNA y su armónico desarrollo socioa fectivo, sino también respecto de las personas con limitaciones en su desempeño cotidiano, por enfermedades, por impedimentos físicos, psíquicos, o por su avanzada edad (Zicavo, 2005).

No basta contar con sala cuna, jardines infantiles de respaldo (por mas imperativa que sea su existencia), la política de desfamiliarización no ha resultado ser eficiente en Chile y tal vez no sea la adecuada para A. Latina. Se deben proteger las familias con políticas públicas que resguarden y cautelen la participación de

\footnotetext{
7. Nota del autor: En condiciones de separación o divorcio tal exaltación pudiera convertirse en un efecto inverso, causante de asimetrías como hemos observado en múltiples oportunidades (Ferrari y Zicavo, 2011).
} 
los individuos en la familia que requiere de aquellos cuidados en su interior; sin castigarlos social y económicamente porque no "contribuyen", tal injusticia no aprecia e invisibiliza su aporte que impide el traspaso a lo social de un asunto no resuelto oportunamente, por la sociedad en su conjunto.

En última instancia "las políticas de desfamiliarización de los cuidados se refieren a transferencias y servicios que delegan las responsabilidades del cuidado desde las familias $-\mathrm{y}$ concretamente desde las mujeres- a servicios con alguna intervención estatal" (Blofield y Martínez, 2014, p. 4).

Blofield y Martínez (2014) citando a Blofield (2012) señalan que:

Por una parte, los oficios domésticos y los cuidados se conciben como una actividad que las mujeres realizan en su condición de mujeres. Al no ser considerado como un verdadero trabajo y, menos aún, un trabajo para el que se requiere calificación, a menudo se estima que su regulación puede prescindir de las normas y límites existentes para otras ocupaciones. Por otra, se entiende que las mujeres que desempeñan estas tareas deben estar incondicionalmente disponibles para atender a las familias de mayores recursos que las contratan. Las propias responsabilidades familiares de las trabajadoras domésticas se esfuman ante su condición de "servidoras (Blofield y Martínez, 2014, p. 16).

Por otra parte se entiende que:

La demanda se concentra, y se refuerza desde un enfoque de derechos, en la necesidad de implementar acciones integrales y no sectoriales $y$ desintegradas. Recuperar el diseño de politicas de corte universal, como imperativo de la aplicación de un enfoque de derechos, es una demanda clara de los propios sistemas de politicas sociales, ya que por más que se siga ampliando la cobertura de programas de transferencias condicionadas $u$ otro tipo de programas dirigidos a la infancia, se sigue menoscabando el ejercicio integral de los derechos de ciudadanía (Pautassi y Royo, 2012, p. 45).

Además, debe tenerse en cuenta que en la medida los ingresos de la familia vayan creciendo, también se verá incrementada la capacidad de traspasar la carga esencial de las labores del hogar a mujeres carenciadas o con ingresos insuficientes que a menudo son contratadas para que realicen dichas tareas, evitando de esta manera los acuerdos con los progenitores para reorganizar las responsabilidades derivadas de la crianza de los NNA. "Por el contrario, cuanto menor es el ingreso, menores son también las opciones de delegar el trabajo doméstico y de cuidados de manera remunerada"(Blofield y Martínez, 2014, p. 7). 


\section{CONCLUSIONES}

En definitiva, el escenario ha sido y parece continuar siendo ineficiente y familiarmente marginador, sobrecargando a la mujer de una función que se insiste le corresponde (al menos ya no explícitamente) y centrifugando al padre de un rol que -de suyo propio- le correspondería asumir, pero del cual es a priori "liberado" o expulsado, marginado por poco útil. Se convierte así en un lugar asumido con algo (o mucho) de comodidad para el hombre y complaciente para la mujer que asume sentirse imprescindible e importante, siendo aprisionada por cadenas "brillantes".

Es indudable que por lo general las políticas públicas y con ello la sociedad, continúan considerando la crianza y el cuidado de NNA (sobre todo de los más pequeños) no como una responsabilidad social, ni siquiera como una responsabilidad de la familia, sino como un compromiso irrenunciable de las mujeres-madres, aunque estemos cerca de que al menos en el discurso se asuma la irresponsabilidad social de tal asumido. Si no es su progenitora, será otra mujer la encargada de la crianza de eseNNA, una hermana, una tía, abuela o empleadas domésticas, profesoras, pero casi siempre una mujer y eso hay que visibilizarlo, es o debe ser ineludible.

Promover la equidad social implica promover los derechos humanos de los NNA, es promover la igualdad de responsabilidades en el presente y futuro, es promover la corresponsabilidad parental; si bien recientes leyes e intenciones políticas en Chile se asoman a ello, aun es una tarea pendiente en su esencia.

Los avances en la conciliación Familia y Estado, entre los tiempos laborales y los familiares, entre las responsabilidades maternas y paternas en el crecimiento y desarrollo de sus hijos e hijas no solo son tímidos, sino que resultan francamente insuficientes. Tensionan una vez más los derechos humanos de las personas a una vida en atención a mayor equidad y simetrías de oportunidades, contradicen las necesidades de protección de los NNA, de su derecho a no ser discriminados, contradicen el derecho a ser escuchados y tenidos en cuenta, a no ser separados de sus familias. Tensionan y limitan el avance social de épocas que hablan de la necesidad del irrestricto respeto a los derechos humanos de las personas, de los adultos mayores, de las mujeres, de los hombres, de las personas con limitaciones sensibles, de las personas enfermas y por supuesto de los NNA.

Se requieren verdaderos compromisos con el cambio y el cambio mismo, con base a políticas eficientes que ejecuten y respeten lo declarado en los convenios ratificados como nación y como sociedad. 


\section{REFERENCIAS}

Andrenacci, L. yRepetto F. (2006). Universalismo, ciudadanía y Estado en la política social latinoamericana. Washington D.C. INDES.Extraído el 12 de Mayo de 2017 desde: https://goo.gl/xiz8VU.

Blofield, M. yMartínez J. (2014). Trabajo, familia y cambios en la política pública en América Latina: equidad, maternalismo y corresponsabilidad. (114), CEPAL. Extraído el 12 de mayo de 2017 desde: https://goo.gl/3XG7RR.

Consenso de Quito (2007). X Conferencia Regional de la Mujer de América Latina y el Caribe. Metas educativas 2021.

Convención sobre los Derechos del Niño, CDN. (1989). Organización de Naciones Unidas. América Latina.

Ferrari, J. y Zicavo, N. (2011). Padres separados, como criar juntos a sus hijos. México: Trillas.

Ley 20.084. Responsabilidad penal adolescente, Chile. Extraído el 09 de mayo de 2017 desde: https://www.leychile.cl.

Muñoz, C. (2013). Proyecto de Ley de Protección Integral de la infancia: ilusión de universalidad. Centro de Políticas Públicas de la Pontificia Universidad Católica. Extraído el 14 de abril de 2017 desde: https:/goo.gl/91CqwD.

Pautassi, L. y Royo, L. (2012). Enfoque de derechos en las politicas de infancia: indicadores para su medición. CEPAL, UNICEF. Extraído el 14 de Abril de 2017 desde: https:// goo.gl/6WgckE

Saavedra, C. (2009). Políticas públicas dirigidas a la infancia y adolescencia. Tensiones y desafíos. Extraído el 04 de mayo de 2017 desde: http://www.uasb.edu.ec/UserFiles/369/ File/PDF/CentrodeReferencia/Temasdeanalisis2/violenciasyderechoshumanos/ saavedra.pdf

Zicavo, N. (2005). Estado y Familia, Carencias y Consecuencias. Psicología para América Latina, (4).Extraído el 12 de Mayo de 2017 desde: https://goo.gl/FT2jjG. 
